\title{
Integrability Conditions for Killing Spinors
}

\author{
P. van Nieuwenhuizen ${ }^{1, \star}$ and N. P. Warner ${ }^{2, \star \star}$ \\ 1 Instituut voor Theoretische Fysica, Princetonplein 5, P.O. Box 80.006, NL-2508 TA Utrecht, \\ The Netherlands \\ 2 California Institute of Technology, Pasadena, CA 91125, USA
}

\begin{abstract}
The conditions for the existence of solutions of $D_{\mu} \eta= \pm c \gamma_{\mu} \eta$ are discussed. In general, it is not sufficient to consider only the first integrability condition $\left[D_{\mu}, D_{v}\right] \eta=-2 c^{2} \gamma_{\mu \nu} \eta$; in particular, the second integrability condition is needed to explain why, in certain cases, only for one choice of sign does a solution exist. The Killing spinor-tensors, as defined by Walker and Penrose, are shown to be the spinorial equivalent of conformal Killing tensors. Their relationship to the Killing spinors and spinor-vectors used in supergravity, is given.
\end{abstract}

In supergravity, Killing spinors are very useful in analyzing the particle spectrum of Kaluza-Klein theories. They also determine the number $N$ of supersymmetries. Killing spinors may be defined [1] to be solutions of

$$
D_{\mu} \eta= \pm c \Gamma_{\mu} \eta,
$$

where $c$ is a constant related to the curvature of the background. The naive integrability condition for this equation $[1,2]$ reads

$$
\left[D_{\mu}, D_{v}\right] \eta=\frac{1}{4} R_{\mu v m n} \Gamma^{m n} \eta=-2 c^{2} \Gamma_{\mu \nu} \eta,
$$

and is clearly insensitive to the sign in (1). It is known that on the round $S_{7}$ there are 8 solutions for either choice of sign, because these solutions have been explicitly constructed [3]. However, on the squashed $S_{7}$, an explicit construction [4] showed that there exists only one solution and for only one choice of sign. Depending on the sign in the Freund-Rubin ansatz $[5,6]$,

$$
F_{\mu \nu \varrho \sigma}= \pm e \varepsilon_{\mu v \varrho \sigma},
$$

\footnotetext{
* On leave from the Institute for Theoretical Physics of the State University of New York at Stony Brook

$\star \star$ Work supported in part by the U.S. Department of Energy under Contract No. DEAC-0381-ER 40050 and Weingart Fellowship
} 
this solution corresponds to $N=0$ or $N=1$ supersymmetry [7]. A similar situation obtains for the $(p, q, r)$-coset spaces of $\mathrm{SU}_{2} \times \mathrm{SU}_{2} \times \mathrm{SU}_{2} / \mathrm{U}(1) \times \mathrm{U}(1)$ [8].

In this article we study the complete set of integrability conditions for (1). The second integrability condition, obtained by differentiating (2) and replacing derivatives of $\eta$ using (1) is sensitive to the sign of $c$, and explains the results described above. Before turning to the integrability of (1), we wish to discuss the notion of Killing spinors and spinor-tensors as introduced by Penrose and Walker (PW) [9], and relate it to the Killing spinors defined by (1) and to the Killing spinor-vectors as introduced by Duff and Pope [1]. The latter are defined by

$$
\bar{D}_{\mu} \eta_{v}+\bar{D}_{v} \eta_{\mu}=0, \quad \bar{D}_{\mu} \equiv \partial_{\mu} \mp c \Gamma_{\mu} .
$$

As we shall show, for spinors both notions coincide, but for vector-spinors the PW notion defines conformal Killing vector-spinors. As with conformal Killing tensors we find, on $S_{n}$, that a conformal Killing vector-spinor is a sum of a lowerspin part $\gamma_{\mu} \lambda$ (analogous to $g_{\mu \nu} C$ for a Killing tensor $T_{\mu \nu}$ ) a derivative of the $k=1$ modes of the Dirac operator, and the ordinary Killing vector-spinors defined in (4).

Penrose and Walker define a spinor $\chi_{B_{1} \ldots B_{s}}^{A_{1}^{\prime} \ldots A_{r}^{\prime}}$ to be a Killing spinor if

$$
\nabla_{\left(B_{0}\right.}^{\left(A_{0}^{\prime}\right.} \chi_{\left.B_{1} \ldots B_{s}\right)}^{\left.A_{1}^{\prime} \ldots A_{r}^{\prime}\right)}=0 \text {. }
$$

Here we are working in $d=4$ dimensions, with a Minkowski metric, and spinor indices $A$ and $A^{\prime}$ take the values 1 or 2 . We will soon, however, move to arbirary $d$. The unsymmetrized derivative on the spinor in (5) will, in general, be an arbitrary linear combination of products of Lorentz spinors with $\varepsilon_{A B}$ or $\varepsilon_{A^{\prime} B^{\prime}}$. Penrose and Walker's Killing equation merely requires that the largest irreducible piece of

$$
\nabla_{B_{0}^{\prime}}^{A_{0}^{\prime}} \chi_{B_{1} \ldots B_{s}}^{A_{1}^{\prime} \ldots A_{r}^{\prime}}
$$

should vanish.

For example, consider a PW Killing vector. It satisfies

$$
\nabla_{(A}^{\left(A^{\prime}\right.} V_{B)}^{\left.B^{\prime}\right)}=0 .
$$

Hence

$$
\nabla_{A}^{A^{\prime}} V_{B}^{B^{\prime}}=\psi_{A B} \varepsilon^{A^{\prime} B^{\prime}}+\psi^{A^{\prime} B^{\prime}} \varepsilon_{A B}+\phi \varepsilon_{A B} \varepsilon^{A^{\prime} B^{\prime}} .
$$

Transcribing into tensor notation and going to arbitrary dimensions one gets

$$
D_{\mu} V_{v}=F_{\mu \nu}+\phi g_{\mu \nu}
$$

where $F_{\mu \nu}=-F_{v \mu}$. Hence

$$
D_{(\mu} V_{v)}=\phi g_{\mu v},
$$

and thus Penrose and Walker's criterion (7) yields a conformal Killing vector.

For a simple spinor $\chi_{B}$, Eq. (5) yields

$$
\nabla_{(A}^{A^{\prime}} \chi_{B)}=0
$$

Hence

$$
\nabla_{A}^{A^{\prime}} \chi_{B}=\lambda^{A^{\prime}} \varepsilon_{A B}
$$


for some spinor $\lambda^{A^{\prime}}$. In tensor notation and generalizing to arbitrary dimensions, one has

$$
D_{\mu} \chi=\gamma_{\mu} \lambda \text {. }
$$

Multiplying by $\gamma^{\mu}$ yields $\lambda$ in terms of $\chi$

$$
D_{\mu} \chi=\frac{1}{n} \gamma_{\mu}(\not D \chi)
$$

Taking the divergence gives

$$
\square \chi=\frac{1}{n} \not D D \chi=\frac{1}{n}\left(\square \chi+\frac{1}{4} R \chi\right),
$$

where $R$ is the Ricci scalar of the manifold. (Note that in our conventions $R<0$ on a sphere.)

Assuming that $R$ is a constant (as, for example, on Einstein spaces) one may use $\not D D \chi=\lambda^{2} \chi$ with $\lambda^{2}=\frac{1}{4} n R(n-1)^{-1}$ and define

$$
\psi_{ \pm}=\chi \pm \lambda^{-1} \not D \chi
$$

to obtain that $D \psi_{ \pm}= \pm \lambda \psi_{ \pm}$. Actually, if the space is Einsteinian,

$$
D_{\mu} \psi_{ \pm}= \pm \frac{\lambda}{n} \gamma_{\mu} \psi_{ \pm}
$$

as one may show as follows. Replace $D_{\mu} \chi$ in $D_{\mu} \psi_{ \pm}$by (14), and evaluate $\lambda^{-1} D_{\mu} \not \chi \chi$ by first commuting the derivative $D_{\mu}$ onto $\chi$ and using (14), and then using $D D \not \chi$ $=\lambda^{2} \chi$ in $\not D \gamma_{\mu} \not D \chi$, one obtains (17). Thus, a PW Killing spinor is a sum of two ordinary Killing spinors.

On $S_{7}$ the spinors in (16) are the $k=0$ modes of the Dirac operator. There are also $8+8$ spinors $\eta_{ \pm}^{I}(I=1,8$; spinor index suppressed) satisfying (17). We may prove, for $S_{7}$, in a simpler way that the $k=0$ modes satisfy (17) by using the completeness relation $\psi_{ \pm}=\sum \eta_{ \pm}^{I}\left(\bar{\eta}_{ \pm}^{I} \psi_{ \pm}\right)$. It is easy to show that $\square\left(\bar{\eta}_{ \pm} \psi_{ \pm}\right)=0$, hence that $\bar{\eta}_{ \pm}^{I} \psi_{ \pm}=$constant. Thus the $k=0$ modes are a linear combination of the $\eta_{ \pm}^{I}$.

We now turn to Killing gravitatinos, but specialize to spheres $S_{n}$ of unit radius. The PW definition (5) in tensor notation generalized to $n$ dimensions, yields

$$
D_{\mu} \chi_{\nu}=A_{\mu \nu}+\gamma_{\mu} \varphi_{v}+\gamma_{\nu} \varphi_{\mu}+g_{\mu \nu} \lambda,
$$

where $A_{\mu v}=-A_{v \mu}$. Hence

$$
D_{(\mu} \chi_{v)}=\gamma_{(\mu} \psi_{v)}+g_{\mu \nu} \lambda
$$

Contracting with $\gamma_{\mu}$ allows us to express $\psi_{\mu}$ in terms of $\gamma \cdot \psi, \lambda$, and $\chi_{\nu}$, and tracing (19) yields $\lambda$ in terms of $\gamma \cdot \psi$ and $\chi_{\nu}$. Remarkably, if one uses these relations to eliminate $\psi_{\mu}$ and $\lambda$ in (19), the $\gamma \cdot \psi$ terms cancel by themselves, yielding

$$
(n+2) D_{(\mu} \chi_{v)}=g_{\mu v} D \cdot \chi+\gamma_{(\mu} D \chi_{v)}+\gamma_{(\mu} D_{v)} \gamma \cdot \chi
$$

Note that (20) is invariant under $\chi_{v} \rightarrow \chi_{v}+\gamma_{v} \xi$. This invariance should be present since the original equation (5) does not make any statement about the components of $\chi$ proportional to $\varepsilon_{A B}$, i.e., to $\gamma_{\mu}$. 
Instead of algebraic operations, one can also use differentiation. Differentiating (20) with $D_{\mu}$ yields

$$
\begin{aligned}
& (n+1) \square \chi_{v}+n D_{v} D \cdot \chi-\gamma_{v} \square \gamma \cdot \chi-\gamma_{v} \not D D \cdot \chi \\
& \quad-D_{v} \not D \gamma \cdot \chi+\frac{5}{4} n(n+1) \chi_{v}-\frac{1}{2}(3 n+2) \chi_{v} \gamma \cdot \chi=0
\end{aligned}
$$

Further differentiation with $D_{v}$ yields

$$
\left[\square+\frac{1}{4}(5 n+4)\right][n D \cdot \chi-\not D \gamma \cdot \chi]=0 .
$$

As a check, note that $\lambda \equiv n D \cdot \chi-\not D \gamma \cdot \chi$ is still invariant under $\chi_{v} \rightarrow \chi_{\nu}+\gamma_{\nu} \xi$. Using $\square \lambda=\left(\not D D-\frac{1}{4} R\right) \lambda$, one notices that $\lambda$ is equal to $\psi_{+}+\psi_{-}$, which are $k=1$ modes of the Dirac operator,

$$
\not D \psi_{ \pm}= \pm i\left(\frac{n}{2}+1\right) \psi_{ \pm} .
$$

The proof is analogous to the discussion following (15).

In order to determine the most general solution of (20), we shift $\chi_{\nu}$ so that $\gamma \cdot \chi=0$. Afterwards we will add to the solution an arbitrary $\gamma_{\mu}$ piece. From (22) we see that

$$
D \cdot \chi=\text { a sum of } k=1 \text { modes of Dirac. }
$$

Introducing the Killing spinor operator

$$
\bar{D}_{\mu} \equiv D_{\mu}-\frac{j}{2} \gamma_{\mu}
$$

where $j= \pm i$, we see that also $\bar{D} \cdot \chi$ is a sum of $k=1$ modes. As suggested by the treatment of conformal Killing vectors, we decompose $\chi_{v}$ into a transversal and longitudinal part

$$
\chi_{v}=\chi_{v}^{T}+\bar{D}_{v} \phi, \quad \bar{D} \cdot \chi^{T}=0 .
$$

(Note the similarity to coexact plus harmonic, and exact forms.) Substituting this decomposition into (20), one obtains

$$
\begin{aligned}
& {\left[(n+2) \delta_{\mu}^{\varrho} \delta_{v}^{\sigma}-\gamma_{\mu} \gamma^{\varrho} \delta_{v}^{\sigma}-\gamma_{v} \gamma^{\varrho} \delta_{\mu}^{\sigma}\right]\left(\bar{D}_{\varrho} \chi_{\sigma}^{T}+\bar{D}_{\sigma} \chi_{\varrho}^{T}\right)} \\
& \quad=-(n+2)\left(D_{\mu} D_{v}+D_{v} D_{\mu}\right) \phi+2\left(D_{\mu} \gamma_{v}+D_{v} \gamma_{\mu}\right) \not D \phi+g_{\mu v}(2 \square \phi+(n-1) \phi) .
\end{aligned}
$$

Note that we have written the left-hand side, L, in terms of $\bar{D}_{\mu}$, but the right-hand side, $\mathrm{R}$, in terms of $D_{\mu}$ because this simplifies (27) greatly.

Our strategy is the same as that usually followed in the case of conformal Killing vectors. We will consider the integral $\int(\overline{\mathrm{L}}-\overline{\mathrm{R}})(\mathrm{L}-\mathrm{R})=0$, and show that $\mathrm{L}$ and $\mathrm{R}$ are orthogonal. Then we will derive the consequences from $\mathrm{L}=0$ and $\mathrm{R}=0$.

To prove the orthogonality of $\mathrm{L}$ and $\mathrm{R}$, we first observe that $g_{\mu \nu}$ acting on $\mathrm{L}$ [the terms on the left-hand side of (27)] yields zero, since it always yields $\left(g^{\varrho \sigma}\right.$ or $\left.\gamma^{\varrho} \gamma^{\sigma}\right)\left(\bar{D}_{\varrho} \chi_{\sigma}^{T}+\bar{D}_{\sigma} \chi_{\varrho}^{T}\right)$, which vanishes due to (26). Partially integrating the remaining terms in $\int \bar{R} L$, one must show that the following expression vanishes

$$
\begin{aligned}
& {\left[(n+2) D^{\mu} D^{\nu}-2 \not D D^{\mu} \gamma^{\nu}\right]} \\
& \quad \cdot\left[(n+2) \delta_{\mu}^{\varrho} \delta_{\nu}^{\sigma}-\left(\gamma_{\mu} \gamma^{\varrho} \delta_{\nu}^{\sigma}+\gamma_{\nu} \gamma^{\varrho} \delta_{\mu}^{\sigma}\right)\right]\left[\bar{D}_{\varrho} \chi_{\sigma}^{T}+\bar{D}_{\sigma} \chi_{\varrho}^{T}\right] .
\end{aligned}
$$


The terms containing $\gamma_{\mu} \gamma^{\varrho} \delta_{v}^{\sigma}+\gamma_{v} \gamma^{\varrho} \delta_{\mu}^{\sigma}$ vanish separately, as one may verify, using

$$
\gamma^{\tau} \gamma_{\sigma}\left[D_{\varrho}, D_{\tau}\right]\left(\bar{D}^{\varrho} \chi_{T}^{\sigma}+\bar{D}^{\sigma} \chi_{T}^{\varrho}\right)=0
$$

and $\mathrm{R}_{\alpha \beta \mu \nu}=\left(g_{\alpha \mu} g_{\beta \nu}-g_{\alpha \nu} g_{\beta \mu}\right)$. In the remaining terms, derivatives and gamma matrices are commuted such that one ends up with only $D \cdot \chi^{T}, \gamma \cdot \chi^{T}, \square$, and $\not D$. ( $\bar{D}$ having been replaced by $\not D-\frac{1}{2} j n$.) Next, one replaces $D \cdot \chi^{T}$ by $\frac{1}{2} j \gamma \cdot \chi^{T}$ according to (26) and (25). The result is that (28) reduces to

$$
-4(n+2)\left[\not D-\frac{1}{2} j n\right]\left[\square+\frac{1}{4}(5 n+4)\right] \gamma \cdot \chi^{T} .
$$

In order to prove the orthogonality of $\mathrm{L}$ and $\mathrm{R}$, it therefore suffices to show that $\gamma \cdot \chi^{T}$ is a zero mode of $\square+\frac{1}{4}(5 n+4)$, or, equivalently, a sum of $k=1$ modes of $\not D$ with eigenvalue $\pm i\left(\frac{n}{2}+1\right)$. Acting on (26) with $\bar{D}^{v}$ and using $\gamma \cdot \chi=0$, one obtains, with (24),

$$
\bar{D}_{v} \bar{D}^{v} \phi=D \cdot \chi=\text { sum of } k=1 \text { modes of } \not D .
$$

The most general solution of this inhomogeneous equation for $\phi$ is a sum of $k=1$ modes (the special solution) and the general solution of $\bar{D}_{v} \bar{D}^{v} \phi_{0}=0$. By considering $\int \bar{\phi}_{0} \bar{D}_{v} \bar{D}^{v} \phi_{0}=0$, it follows that $\bar{D}_{v} \bar{D}^{v} \phi_{0}=0$ implies $\bar{D}_{v} \phi_{0}=0$. Hence, $\phi_{0}$ is either $\eta_{+}$

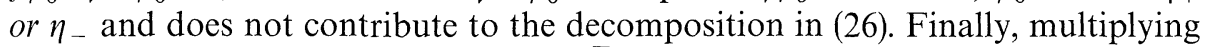
now (26) by $\gamma^{v}$, one sees that $\gamma \cdot \chi^{T}=-\bar{D} \phi=(k=1)$ modes of $\not D$. Orthogonality is proven. Therefore, the left- and right-hand sides of (27) vanish independently.

To deduce the consequences of $\mathrm{L}=0$ and $\mathrm{R}=0$, we begin by contracting the left-hand side of (27) with $D^{\mu}$. After some algebra, one finds

$$
(n+1)\left(\square \chi_{\sigma}^{T}+\frac{1}{4} 5 n \chi_{\sigma}^{T}\right)=\left(D_{\sigma}+\frac{1}{2} j \gamma_{\sigma}\right) \bar{D} \gamma \cdot \chi^{T} .
$$

As a check one may verify that, upon further contraction with $\gamma^{\sigma}$, one recovers the result that $\gamma \cdot \chi^{T}$ is a zero mode of $\square+\frac{1}{4}(5 n+4)$. As we already discussed, this implies that $\gamma \cdot \chi^{T}$ is a sum of $k=1$ modes of Dirac. Hence, the right-hand side of (32) is of the form $D_{\sigma}$ or $\gamma_{\sigma}$ times $k=1$ modes of $\not D$. The latter we denote again by $\phi^{+}$ and $\phi^{-}$. Assuming that $\chi_{\sigma}^{T}$ has the same form as the right-hand side of (32), one finds the following particular solution of (32)

$$
\begin{aligned}
\chi_{\sigma}^{T}(\operatorname{part})= & \alpha_{+}\left(D_{\sigma}-\frac{1}{2}(n+2) i \gamma_{\sigma}-\frac{1}{2}(n-1) j \gamma_{\sigma}\right) \varphi_{+} \\
& +\alpha_{-}\left(D_{\sigma}+\frac{1}{2}(n+2) i \gamma_{\sigma}-\frac{1}{2}(n-1) j \gamma_{\sigma}\right) \varphi_{-} .
\end{aligned}
$$

Note the symmetry under $i \rightarrow-i$. This particular solution satisfies

$$
\begin{aligned}
D \cdot \chi^{T}(\text { part }) & =\frac{j}{2} \gamma \cdot \chi^{T}(\text { part }) \\
& =\frac{1}{4}(n-1)\left[\alpha_{+}(n-i j(n+2)) \varphi_{+}+\alpha_{-}(n+i j(n+2)) \varphi_{-}\right] .
\end{aligned}
$$

Hence, $\bar{D} \cdot \chi^{T}$ (part) $=0$.

Let us fix $\alpha_{+}$and $\alpha_{-}$such that $\gamma \cdot \chi^{T}$ (part) $)=\gamma \cdot \chi^{T}$. Defining $\theta_{\sigma}$ by $\chi_{\sigma}^{T}=\chi_{\sigma}^{T}$ (part) $+\theta_{\sigma}$, it follows that $\bar{D} \cdot \theta=\gamma \cdot \theta=D \cdot \theta=0$. It also follows from (32) that $\theta_{\sigma}$ is a zero mode of $\square+\frac{1}{4} 5 n$. Hence, $\not D D \theta_{\sigma}=-\frac{1}{4}(n+2)^{2} \theta_{\sigma}$ and decomposing $\theta_{\sigma}$ into $\theta_{\sigma}=\theta_{\sigma}^{+}+\theta_{\sigma}^{-}$as before, it follows that $\not D \theta_{\sigma}^{ \pm}= \pm \frac{i}{2}(n+2) \theta_{\sigma}^{ \pm}$. 
Since the eigen-modes of $\not D$ on $\psi_{\sigma}$ with $\gamma \cdot \psi=D \cdot \psi=0$ are given by $\lambda= \pm \frac{i}{2}(n+2+k)$, we conclude that $\theta_{\sigma}$ is a sum of $k=0$ modes of $\not D$. Using the completeness of the $\eta_{ \pm}^{I}$, we note that $\theta_{\sigma}^{ \pm}=\sum \eta_{ \pm}^{I} \phi_{ \pm \sigma}^{I}$, where the $\phi_{ \pm \sigma}^{I} \equiv \bar{\eta}_{ \pm}^{I} \theta_{\sigma}^{ \pm}$satisfy $\square \phi_{\sigma}=-(n-1) \phi_{\sigma}$, and thus are Killing vectors. Indeed, $\square \eta_{ \pm}=-\frac{n}{4} \eta_{ \pm}$and $\square \theta_{\sigma}^{ \pm}$ $=-\frac{1}{4} 5 n \theta_{\sigma}^{ \pm}$, while $\left(D_{\mu} \bar{\eta}_{+}^{I}\right)\left(D^{\mu} \theta_{\sigma}^{+}\right)=\frac{1}{4}(n+2) \bar{\eta}_{+}^{I} \theta_{\sigma}$.

The general solution of (32) is thus that $\chi_{\sigma}^{T}$ is the sum of (33) and of $\theta_{\sigma}$. To find the general solution of $\mathrm{L}=0$ in (27), we first substitute $\theta_{\sigma}$ into $\mathrm{L}$. The result is $\left(D_{\mu} \mp \frac{1}{2} i \gamma_{\mu}\right) \theta_{v}^{ \pm}+(\mu \leftrightarrow v)=0$. This equation is indeed satisfied, because $\theta_{v}^{ \pm}$are a product of $\eta_{ \pm}^{I}$ and Killing vectors. Thus, $\theta_{\sigma}^{+}$and $\theta_{\sigma}^{-}$are ordinary Killing vectorspinors. They contain the obvious solution $\bar{D}_{\mu} \chi_{\nu}^{T}+\bar{D}_{\nu} \chi_{\mu}^{T}=0$ of (27).

We next substitute (33) into $\mathrm{L}=0$. Now $\varphi_{ \pm}=\sum \eta_{ \pm}^{I} \phi_{ \pm}^{I}$, where $\phi_{ \pm}^{I}=\sum \bar{\eta}_{ \pm}^{I} \varphi_{ \pm}$are $k=1$ modes of $\square$, which can also be written as $\bar{\eta}_{+}^{I} \eta_{-}^{J}$. (There are only $n+1$ independent $k=1$ modes. On $S_{7}$ they are obtained by contracting $\bar{\eta}_{+}^{I} \eta_{-}^{J}$ - with either $\delta_{I J}$ or $\gamma_{I J}^{\sigma}$.) It follows that the gradient of the scalar $k=1$ mode $\phi$ is a conformal Killing vector, $D_{\mu} D_{\nu} \phi=-g_{\mu \nu} \phi$, and using this information, one may check that also $\chi_{v}^{T}$ (part) in (33) satisfies (27). (The $\gamma_{(\mu} D_{v)} \varphi_{ \pm}$and $g_{\mu v} \varphi_{ \pm}$terms cancel separately.)

To deduce the consequences of the vanishing of $\mathrm{R}$, the right-hand side of (27), we note that $\mathrm{R}$ vanishes identically when contracted with $g_{\mu \nu}$ or $\gamma_{\mu}$, but contraction of $\mathrm{R}$ with $D_{\mu}$ yields a nonvanishing result

$$
-2\left[n D_{v}-\gamma_{v} \not D\right]\left[\square \phi+\frac{1}{4}(5 n+4) \phi\right]=0 .
$$

The general solution is a sum of $k=1$ modes of $\not D$ [the general solution of $\square \phi$ $\left.+\frac{1}{4}(5 n+4) \phi=0\right]$ plus any $k=0$ mode of $\not D$ (conformal Killing spinors were ordinary Killing spinors as we saw). This information on $\phi$ is slightly weaker than that obtained below (31) since there we showed that only $\eta_{+}$or $\eta_{-}$could be present.

This concludes our discussion of the PW Killing spinor-tensors. We have shown that on Einstein spaces PW Killing spinors coincide with the ordinary Killing spinors of supergravity, but that PW Killing vector-spinors on $S_{n}$ are a sum of (i) $\gamma^{\mu}$ times an arbitrary spinor, (ii) a sum of ordinary Killing vector-spinors, and (iii) the gradient of a $k=1$ Dirac mode. of (1).

We now turn to the second part of our investigation, namely the integrability

It is clear that the first integrability condition (2) is necessary for a solution of (1) to exist. However, by differentiating (2) and using (1) to substitute for $D_{\mu} \eta$, one may derive a second integrability condition. Moreover, by doing further differentiations and substitutions, one can obtain an infinite set of necessary algebraic constraints on $\eta$. A sufficient condition for the existence of a solution to (1) has been given by Schouten [10]: if the $(n+1)^{\text {th }}$ integrability condition derived in the manner described above, is satisfied as a consequence of the first $n$ conditions, then these first $n$ conditions are sufficient for a solution of (1) to exist.

In fact, matters are even simpler than one might think. The first (naive) integrability condition (2) states that inside the hypersurface defined by the vectors $\partial_{\mu} \eta(x)$ in $(\eta, x)$ space, the curvature vanishes. Hence, in that hypersurface, one can integrate. The problem is to make sure that one does not leave the hypersurface, while integrating. This is the content of the higher order integrability conditions 
and all one has to do is to find the $n$ for which the $n+1^{\text {th }}$ integrability condition has the same solutions as the $n^{\text {th }}$ condition. If this is the case, one can stop at this point and integrability holds [11].

In supergravity, the stability subgroup of $\mathrm{SO}(7)$ for one spinor is $G_{2}$, for two spinors it is $\mathrm{SU}_{3}$ for three spinors it is the same as for four spinors, namely $\mathrm{SU}_{2}$, while 5 or more spinors have trivial stabilizer. Thus the solution space is at most 4-dimensional (with the exception of the round $S_{7}$ for which it is 8-dimensional). Therefore, one has to check at most five integrability conditions.

By multiplying (2) by $\gamma^{v}$, one may show that

$$
\left[R_{\mu \nu}-4 c^{2}(n-1) g_{\mu \nu}\right] \gamma^{v} \eta=0 .
$$

Multiplying (35) by $\eta^{+} \gamma^{\varrho}$ and then adding the result to its complex conjugate, one obtains

$$
\left[R_{\mu \nu}-4 c^{2}(n-1) g_{\mu v}\right]\left(\eta^{+} \gamma^{o} \gamma^{v} \eta+\eta^{+} \gamma^{v} \gamma^{o} \eta\right)
$$

and hence

$$
R_{\mu v}=4 c^{2}(n-1) g_{\mu v} .
$$

Therefore, the existence of a Killing spinor implies that the space is Einstein.

Using (37), the integrability condition (2) may be rewritten more succinctly as [2]

$$
C_{\mu \nu \varrho \sigma} \gamma^{\varrho \sigma} \eta=0 \text {, }
$$

where $C_{\mu \nu \rho \sigma}$ is the Weyl tensor.

The second integrability condition is obtained by differentiating (38) and using (1)

$$
\partial_{\lambda} C_{\mu \nu \varrho \sigma} \gamma^{\varrho \sigma} \eta=-C_{\mu \nu \varrho \sigma} \gamma^{\varrho \sigma} \partial_{\lambda} \eta=\left(-C_{\mu \nu \varrho \sigma} \gamma^{\varrho \sigma}\right)\left(-\frac{1}{4} \omega_{\lambda m n} \gamma^{m n} \pm c \gamma_{\lambda}\right) \eta
$$

Using (38) this may be rewritten in a manifestly covariant form

$$
\left(D_{\lambda} C_{\mu \nu \varrho \sigma}\right) \gamma^{\varrho \sigma} \eta \mp 2 c C_{\mu \nu \lambda \varrho} \gamma^{\varrho} \eta=0 .
$$

If the Einstein space is a symmetric space (for which $D_{\lambda} R_{\mu \nu \varrho \sigma}=0$ ), it follows from the same arguments used to derive (37) that $C_{\mu \nu \lambda \varrho}=0$, and hence the manifold must be a maximally symmetric space (a sphere or a sphere factored by some finite group). On the other hand, if the space is not a symmetric space, both terms in (40) are nonvanishing and the null space $N$ of spinors satisfying (38) decomposes into three disjoint subspaces $N_{+}, N_{-}$, and $N_{0}$, where $N_{+}\left(N_{-}\right)$satisfies (40) with $-(+)$ sign, and $N_{0}$ does not satisfy either case. (If one given $\eta$ lies in both $N_{+}$and $N_{-}$, then $C_{\mu \nu \rho \sigma}=0$.) This explains why on the squashed $S_{7}$, for which $N$ is onedimensional, only one choice of sign in (1) is allowed. From explicit computations it is known that for one of the $(p, q, r)$ spaces of $G / H=\mathrm{SU}_{2} \times \mathrm{SU}_{2} \times \mathrm{SU}_{2} / \mathrm{U}_{1} \times \mathrm{U}_{1}$, (38) does have a solution, in fact, it has in that case precisely two solutions. Both of these have been shown to correspond to solutions of (1) with the same choice of sign. This result could also be deduced from (38) and (40). One would find that these conditions are satisfied for only one choice of sign, and that for that choice of sign, both solutions of (38) are solutions of (40). Since the dimension of the solution space of (38) is not decreased by (40), it follows that Eq. (1) is integrable and has two solutions.

This concludes our discussion of the integrability conditions of (1). We now end with a brief discussion on the integrability condition of the conformal Killing 
spinors in (14) when one does not assume, as we did, that the space is Einsteinian. By evaluating $\left[D_{\mu}, D_{\nu}\right] \chi$ and commuting in $D_{\mu} \not \chi_{\chi}$ the $D_{\mu}$ onto $\chi$, we again use (14) and eliminate the $\square$ on $\chi$ by (15). Surprisingly, all terms add up to (38). In other words, a conformal Killing spinor of a non-Einsteinian space still must lie in the null-space of the de Sitter holonomy group.

Acknowledgement. It is a pleasure to thank B. Allen for discussions.

\section{References}

1. Duff, M.J., Pope, C.N.: In: Supersymmetry and supergravity ' 82, S. Ferrara, J. G. Taylor, and P. van Nieuwenhuizen (eds.). Singapore: World Publ. Co. 1983

2. Englert, F., Rooman, M., Spindel, Ph.: Supersymmetry breaking by torsion and the Ricci-flat squashed seven spheres. Phys. Lett. 127 B, 47 (1983)

3. D'Auria, R., Fré, P., Nieuwenhuizen, P. van: Symmetry breaking in $d=11$ supergravity on the parallelized seven-sphere. Phys. Lett. 122 B, 225 (1983)

4. Awada, M.A., Duff, M.J., Pope, C.N.: $N=8$ supergravity breaks down to $N=1$. Phys. Rev. Lett. 50, 294 (1983)

5. Freund, P.G.O., Rubin, M.A.: Dynamics of dimensional reduction. Phys. Lett. 97 B, 233 (1980)

6. Castellani, L., Warner, N.P.: The SO (7) symmetry of $d=11$ supergravity on the parallelized seven-sphere. Phys. Lett. 130 B, 47 (1983)

7. Duff, M.J., Nilsson, B., Pope, C.N.: Spontaneous supersymmetry breaking by the squashed seven-sphere. Phys. Rev. Lett. 50, 2043 (1983)

8. D'Auria, R., Fré, P., Nieuwenhuizen, P. van: $N=2$ matter coupled supergravity on a coset $G / H$ possessing an additional Killing vector. Utrecht preprint 1983

9. Walker, M., Penrose, R.: On the quadratic first integrals of the geodesic equations for type $\{22\}$ spacetimes. Commun. Math. Phys. 18, 265 (1970)

10. Schouten, J.A.: Ricci calculus. Berlin, Heidelberg, New York: Springer 1956, pp. 80-81

11. Duistermaat, H.: Private communication

Communicated by S. W. Hawking

Received October 7, 1983

Note added in proof. Following the completion of this paper, we learned of two pieces of work which have some bearing on our results.

First, Castellani and Romans (" $N=3$ and $N=1$ Supersymmetry in a New Class of Solutions for $d=11$ Supergravity", Caltech preprint CALT-68-1053) have shown that the higher order integrability conditions can provide non-trivial constraints on the solution space. In particular, Eq. (38) is shown to have four solutions; whereas the Killing spinor equation (1) can, depending upon the geometry, have either one or three solutions for one sign and no solutions for the other sign.

Duff, Nilsson, and Pope ("The Criterion for Vacuum Stability in Kaluza-Klein Supergravity," Imperial College preprint /83-84/18) have shown that for Einstein manifolds with $\Lambda=0$, the Killing spinor equation has solutions $\eta_{+}$and $\eta_{-}$[with opposite signs in (1), respectively], if and only if the manifold is a sphere, $S^{n}$, for $n>2$. Consequently, if one is not on a round sphere, the solution space of (1) must be empty for one choice of sign. The proof is as follows. Consider the scalar, $\phi=\bar{\eta}_{+} \eta_{-}$and the vector $V_{a}=\bar{\eta}_{+} \Gamma_{a} \eta_{-}$. If $\phi \not \equiv 0$, then it satisfies $\nabla_{a} \nabla_{b} \phi$ $=-g_{a b} \phi$, and its gradient is a conformal Killing vector. Yono and Nagano [Ann. Math. 69, 451 (1959)] have shown that such scalars can only exist on round spheres $(n>2)$. If $\phi=0$ then $V_{a} \not \equiv 0$, but $\nabla_{b} V_{a} \equiv 0$. Hence $\nabla_{[a} V_{b]} V_{a} \equiv 0$, and thus $R_{a b c d} V^{d}=0$, which implies $R_{b d} V^{d}=\Lambda V_{b}=0$. Therefore, $\Lambda=0$. 